INPLASY PROTOCOL

To cite: Zhang et al. Chinese Patent Medicine Shufeng Jiedu Capsule for Acute Upper Respiratory Tract Infections: A Protocol of a Systematic Review of Randomized Clinical Trials. Inplasy protocol 202050083. doi:

10.37766/inplasy2020.5.0083

Received: 22 May 2020

Published: 22 May 2020

Corresponding author:

Ying-ying Zhang

yuchen8240@126.com

Author Affiliation:

Centre for Evidence-Based

Chinese Medicine

Support: The UK and Chinese governments

Review Stage at time of this submission: Preliminary searches.

\section{Chinese Patent Medicine Shufeng Jiedu Capsule for Acute Upper Respiratory Tract Infections: A Protocol of a Systematic Review of Randomized Clinical Trials}

Zhang, YY1; Xia, RY2; Liang, SB37 Dai, MY4; Hu, XY5; Willcox, M6; Moore, M77; Fei YT8; Liu, JP9.

Review question / Objective: A systematic review and metaanalysis will be conducted to evaluate whether Chinese patent medicine Shufeng Jiedu capsule (SFJD) is effective in relieving symptoms, shortening the course, and influencing the use of antibiotics or antivirals in acute upper respiratory tract infections (AURTIs). Parallel group, randomized controlled trials in regardless of blinding will be included. SFJD capsule is used alone or in combination with symptomatic treatment or/and etiological treatment for AURTIs, including common cold, acute pharyngitis, acute laryngitis, herpangina, pharyngoconjunctival fever, acute rhinitis, or acute tonsillitis.

INPLASY registration number: This protocol was registered with the International Platform of Registered Systematic Review and Meta-Analysis Protocols (INPLASY) on 22 May 2020 and was last updated on 22 May 2020 (registration number INPLASY202050083).

\section{INTRODUCTION}

Review question / Objective: A systematic review and meta-analysis will be conducted to evaluate whether Chinese patent medicine Shufeng Jiedu capsule (SFJD) is effective in relieving symptoms, shortening the course, and influencing the use of antibiotics or antivirals in acute upper respiratory tract infections (AURTIs). Parallel group, randomized controlled trials in regardless of blinding will be included. SFJD capsule is used alone or in 
combination with symptomatic treatment or/and etiological treatment for AURTIs, including common cold, acute pharyngitis, acute laryngitis, herpangina, pharyngoconjunctival fever, acute rhinitis, or acute tonsillitis.

Condition being studied: Upper respiratory tract infections (URTIs) are the most commonly occurring diseases. In 2017 , the global incidence of upper respiratory infections was $\mathbf{1 7 . 1}$ billion worldwide. It is reported that acute upper respiratory tract infections (AURTIs) are the primary cause of patients seeking medical care in the United States, and as high as $75 \%$ of antibiotic prescriptions are for the treatment of AURTIs in high-income countries. However, AURTIs are predominantly caused by virus and are selflimited disease. Based on existing evidence, guidelines recommend that antibiotics should not be prescribed for patients with AURTIs without serious infection indications, and symptomatic treatments are recommended. In addition, complementary treatments should also be considered to alleviate persistent symptoms for AURTIs. A published systematic review suggests that traditional Chinese medicine plays an important role in shortening the natural course and improving symptoms of URTIs. SFJD capsule is an oral patent Chinese herbal medicine which has been widely used in China for the treatment of respiratory infections for $\mathbf{3 0}$ years. An in vitro study of antibacterial testing suggested that SFJD had a broad-spectrum antibacterial effect. This systematic review on randomized controlled trials will evaluate the therapeutic effect and safety of SFJD capsule for AURTIs.

\section{METHODS}

Search strategy: We comprehensively search randomized controlled trials from PubMed, the Cochrane Library, EMBASE, Scopus, Web of Science, China National Knowledge Infrastructure (CNKI), Chinese Scientific Journal Database (VIP), SinoMed and Wanfang database from the establishment of the database to May,
2020. A systematic search is also conducted of Clinical Trials. gov (www.ClinicalTrials.gov) and Chinese Clinical Trial Registry (http:// www.chictr.org.cn/index.aspx). An example of a search strategy for PubMed: ((((Shufengjiedu[Title/Abstract]) OR Shu Feng Jie Du[Title/Abstract]) OR Shu-FengJie-Du[Title/Abstract]) OR Shufeng Jiedu[Title/Abstract]) OR ShufengJiedu[Title/Abstract].

Participant or population: Patients (adults and children) with clinical diagnose of AURTIs regardless of gender, age or ethnics from primary care or outpatient department of hospitals.

Intervention: Chinese patent medicine SFJD capsule is used alone or in combination with symptomatic treatment (antipyretic, decongestant) or/and etiological treatment (antiviral, antibacterial treatment) as interventions.

Comparator: Placebo, no treatment, symptomatic treatment or/and etiological treatment.

Study designs to be included: Randomized controlled trials.

Eligibility criteria: Study type: we will include only parallel group, randomized controlled trials in regardless of blinding; study population: adults or children diagnosed with AURTIs in regardless of gender, age or ethnics from primary care or outpatient department of hospitals; intervention: the eligible intervention including SFJD capsule used alone or in combination with symptomatic treatment (antipyretic, decongestant) or/and etiological treatment (antiviral, antibacterial treatment); comparators: the eligible comparators including placebo, no treatment, symptomatic treatment or/and etiological treatment for AURTIs; outcomes: the primary outcome will be symptom relief measured by resolution time of typical respiratory symptoms, the additional outcomes including: (1) cure rate; (2) symptom improvement rate; (3)adverse events. 
Information sources: We comprehensively search trials from PubMed, the Cochrane Library, EMBASE, Scopus, Web of Science, China National Knowledge Infrastructure (CNKI), Chinese Scientific Journal Database (VIP), SinoMed and Wanfang database. A systematic search is also conducted in Clinical Trials.gov (www.ClinicalTrials.gov) and Chinese Clinical Trial Registry (http:// www.chictr.org.cn/index.aspx). There is no language limit.

Main outcome(s): The primary outcome is symptom relief measured by resolution time of typical respiratory symptoms (such as catarrh symptoms, fever and sore throat in patients with common cold; sore throat and dry throat in patients with acute pharyngitis; sore throat, herpes and fever in patients with herpangina; fever, sore throat and conjunctivitis in patients with pharyngoconjunctival fever; sore throat, swollen tonsils and fever in patients with acute tonsillitis; stuffy nose and runny nose in patients with acute rhinitis); The outcomes will be measured within 5 days of starting treatment.

Additional outcome(s): Cure rate; symptom improvement rate; adverse events.

Data management: After removing duplicates, two review authors will independently screen studies by titles and abstracts; uncertainty and insufficient information will be determined for eligibility through obtaining full texts, and disagreement will be resolved by discussion to reach a consensus. In the full text screening process, reasons for excluded studies will be recorded. A predefined data extraction form will be used by two review authors to independently extract data, including author, year, title, study population, interventions, comparisons, measurements, outcomes, as well as study characteristics (sample size, setting and funding). Discrepancies will be resolved by discussion or determined by a third-party adjudication if necessary.
Quality assessment / Risk of bias analysis: The quality assessment of individual included trials will be performed and verified successively by two authors using a revised Cochrane Risk of Bias Tool. This modified tool proposes to reduce the number of "unclear" responses and provides four response options: "probably no", "no", "probably yes", and "yes". The method of GRADE (Grading of Recommendations Assessment, Development and Evaluation) will be employed to rate the certainty of synthesized evidence of pre-defined outcomes in five aspects (risk of bias, directness, precision, consistency, and the possibility of publication bias). Disagreements will be resolved by discussion or determined by a third-party adjudication if necessary.

Strategy of data synthesis: For dichotomous data, we will calculate risk ratio (RR) with $95 \%$ confidence intervals (CI); for continuous data, we will calculate mean difference (MD) or standard mean difference (SMD) with 95\% Cl. Cochrane Review Manger 5.3 software will be employed in data analysis process. We will synthesize the data quantitatively when the included trials have acceptable clinical homogeneity. Cochrane $Q$ test and $I^{2}$ statistic will be employed to assess statistical heterogeneity, and we will also combine the data quantitatively when the heterogeneity can be explained reasonably by predefined subgroup analysis. Otherwise we will describe the results narratively. A fixed-effects model will be considered when $\mathrm{I} 2$ is $<30 \%$, otherwise, a random-effects model will be used. Funnel plot will be employed to test publication bias when there are more than ten eligible studies of any one outcome.

Subgroup analysis: To explain heterogeneity, we will predefine subgroup analysis via: the severity of AURTIs; the age of patients (adults, children $(2 \leq$ age $\leq 14)$ ).

Sensibility analysis: When the primary outcomes have clinically meaningful differences, sensitivity analysis will be employed to challenge the robustness of 
the results in following methodological aspects: comparison between clear randomization concealment and unclear randomization concealment; comparison between reported loss- to-follow-up and not reported.

Language: There is no language limit.

Country(ies) involved: China and UK.

Other relevant information: Support: This work is supported by the National Key Research and Development Project (grant no. 2018YFE0102300) and Innovate UK (grant no. 104287-610239). Ru-yu Xia, Meng-yuan Dai, Yu-tong Fei, and Jian-ping Liu, received this research funding from Ministry of Science and Technology of the People's Republic of China. Xiao-yang $\mathrm{Hu}$, Merlin Willcox, and Michael Moore received this research funding from Innovate-UK.

Keywords: Chinese patent medicine; Shufeng Jiedu; acute upper respiratory tract infections; randomized clinical trials; systematic review.

Contributions of each author:

Author 1 - Ying-ying Zhang - Author 1 conceived the review and will carry out the study.

Author 2 - Ru-yu Xia - Author 2 conceived the review and participated in the study design.

Author 3 - Shi-bing Liang - Author 3 participated in the study design.

Author 4 - Meng-yuan Dai - Author 4 participated in the study design.

Author 5 - Xiao-yang Hu - Author 5 participated in the study design.

Author 6 - Merlin Willcox - Author 6 revised the draft protocol.

Author 7 - Michael Moore - Author 7 revised the draft protocol.

Author 8 - Yu-tong Fei - Author 8 revised the draft protocol.

Author 9 - Jian-ping Liu - Author 9 conceived the review and provided substantial feedbacks in the study design. Jian-ping Liu is the corresponding author.

Conflicts of interest: The authors declare that they have the following possible conflicts of interest. However, these conflicts of interest do not actually influence the design, analyses, and the reporting of this study. This work is supported by the project investigating the use of Chinese herbal medicine for the treatment of chronic obstructive pulmonary disease. The project is part of the "UKChina collaboration to tackle antimicrobial resistance", funded by the UK and Chinese governments. 